\title{
Reconfigurable Charge Pump Circuit with Variable Pumping Frequency Scheme for Harvesting Solar Energy under Various Sunlight Intensities
}

\author{
Jeong Heon Kim, Sang Don Byeon, Hyun-Sun Mo, and Kyeong-Sik Min \\ School of Electrical Engineering, Kookmin University, Seoul 136-702, Republic of Korea \\ Correspondence should be addressed to Kyeong-Sik Min; mks@kookmin.ac.kr
}

Received 13 November 2013; Revised 1 March 2014; Accepted 1 March 2014; Published 15 April 2014

Academic Editor: Ismail H. Altas

Copyright (C) 2014 Jeong Heon Kim et al. This is an open access article distributed under the Creative Commons Attribution License, which permits unrestricted use, distribution, and reproduction in any medium, provided the original work is properly cited.

\begin{abstract}
We propose variable pumping frequency (VPF) scheme which is merged with the previous reconfigurable charge pump (RCP) circuit that can change its architecture according to a given sunlight condition. Here, merging the VPF scheme with the architecture reconfiguration can improve percentage output currents better by $21.4 \%$ and $22.4 \%$ than RCP circuit with the fixed pumping frequencies of $7 \mathrm{MHz}$ and $15 \mathrm{MHz}$, respectively. Comparing the VPF scheme with real maximum power points (MPP), the VPF can deliver $91.9 \%$ of the maximum amount of output current to the load on average. In terms of the power and area overheads, the VPF scheme proposed in this paper consumes the power by $0.4 \%$ of the total power consumption and occupies the layout area by $1.61 \%$ of the total layout area.
\end{abstract}

\section{Introduction}

Solar energy is one of the most popular energy sources that can be harvested from environment because of its ubiquitous availability and high power density, and that DC voltage and current can be directly obtained from sunlight [1-3]. Due to these advantages that are mentioned just earlier, harvesting solar energy becomes more popular and useful in various applications such as wireless sensor networks (WSN) $[4,5]$. Among various solar energy harvesting systems, in this paper, we consider a simple capacitor-based charge pump circuit for harvesting a small amount of solar energy $[6,7]$. The charge pump circuit with small-scale energy harvesting can be implemented with low cost and simple architecture; thus, it can be suitable to tiny low-power and low-cost systems such as WSN systems.

One problem in developing solar energy harvesting systems is that sunlight is not static but changes dynamically. As a result of this dynamic variation of sunlight, an amount of solar energy that can be converted to electrical voltage and current by energy harvesting systems can be changed. When sunlight is very strong, the amount of solar energy that can be converted to electrical voltage and current is large. On the contrary, for weak sunlight, the amount of solar energy is small; thereby, the converted amounts of voltage and current become small, too. Figure 1(a) shows the current-voltage relationship of solar cell for various sunlight conditions. For the sunlight intensity as strong as $38 \mathrm{~mA}$, maximum power point (MPP) that means a bias point that can deliver maximum amount of current-voltage product of solar cell is given at $V_{\mathrm{SC}}=2.97 \mathrm{~V}$ and $I_{\mathrm{SC}}=32.7 \mathrm{~mA}$. Here $V_{\mathrm{SC}}$ and $I_{\mathrm{SC}}$ are the solar cell's voltage and current, respectively. If the sunlight intensity becomes as small as $2 \mathrm{~mA}$ in weak sunlight condition, the MPP moves to $V_{\mathrm{SC}}=1.81 \mathrm{~V}$ and $I_{\mathrm{SC}}=1.61 \mathrm{~mA}$, as shown in Figure 1(a). Figure 1(b) shows the relationship of solar cell's voltage and power, where the solar cell's power is calculated with the product of $V_{\mathrm{SC}}$ and $I_{\mathrm{SC}}$. One more thing to note here is that the sunlight intensity should be expressed by lumen or candela unit. In this paper, however, we expressed the sunlight intensity by amount of solar cell's photocurrent, because the solar cell's photocurrent is directly depending on the sunlight intensity [8]. Instead of using lumen or candela, the solar cell's photocurrent 


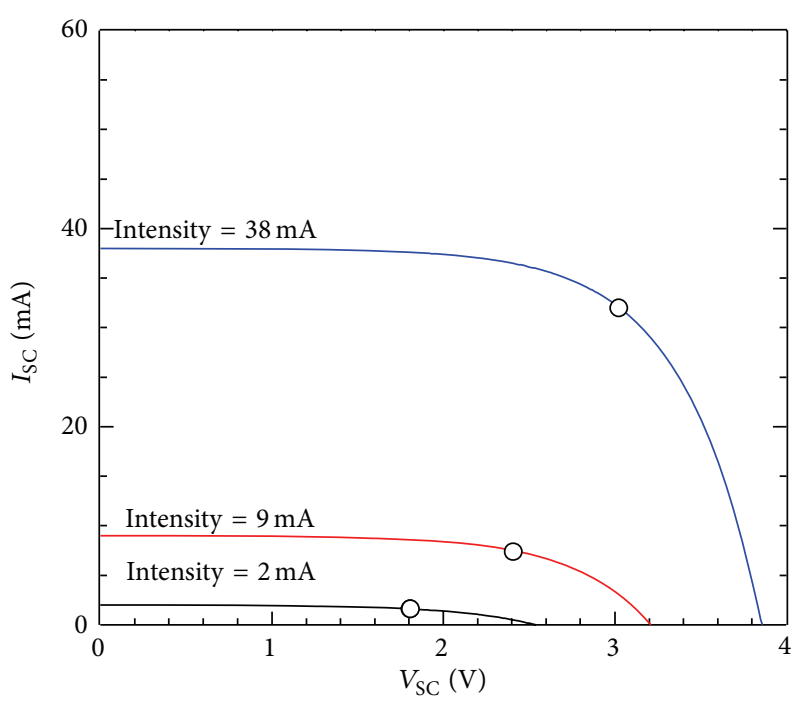

O Maximum power point

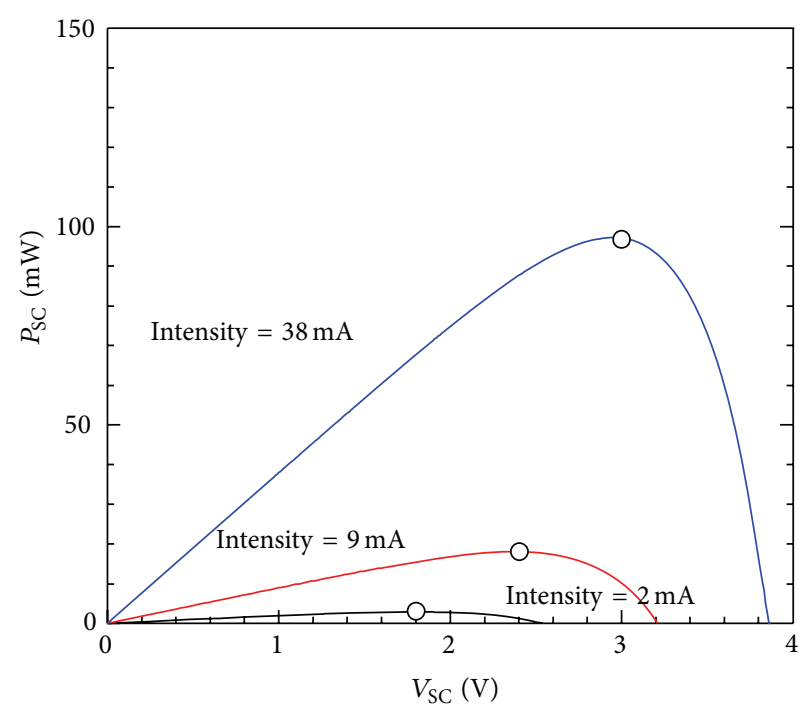

Maximum power point

(a)

(b)

FIGURE 1: (a) Relationship of solar cell's current $\left(I_{\mathrm{SC}}\right)$ and voltage $\left(V_{\mathrm{SC}}\right)$ according to sunlight intensity variation. (b) Relationship of solar cell's power $\left(P_{\mathrm{SC}}\right)$ and voltage $\left(V_{\mathrm{SC}}\right)$ according to sunlight intensity variation.

for representing sunlight intensity can make us easier in understanding the energy harvesting system.

Lee et al. proposed a reconfigurable charge pump (RCP) circuit that can adjust the architecture among the serial, the parallel-serial, and the parallel modes according to sunlight variation to deliver the largest amount of power to the output at a given sunlight condition [9]. The conceptual diagrams of operation of RCP circuit are shown in Figures 2(a), 2(b), and 2(c), where weak, moderate, and strong sunlight conditions are illustrated, respectively. In Figure 1(a), we can know that $V_{\mathrm{SC}}$ and $I_{\mathrm{SC}}$ in weak sunlight are smaller than moderate and strong sunlight conditions. In Figure $2(\mathrm{a}) V_{\mathrm{SC}}$ in weak sunlight is as low as $1.81 \mathrm{~V}$ which is about $71.3 \%$ of the opencircuit voltage $\left(V_{\mathrm{OC}}\right)$ in Figure 1(a). On the contrary, the output voltage, $V_{\mathrm{BAT}}$, should be the same with the charging voltage of lithium ion batteries, that is, as high as $4.2 \mathrm{~V}$. Thus the number of stages of charge pump's architecture should be large enough to generate $4.2 \mathrm{~V}$ from $1.81 \mathrm{~V}$.

One more thing to consider here is that an amount of pumping current should be small in weak sunlight because the solar cell can deliver only small amounts of $V_{\mathrm{SC}}$ and $I_{\mathrm{SC}}$ to the load. Here we can think that the serial architecture of charge pump in Figure 2(a) is more suitable than the parallelserial and parallel architectures that are shown in Figures 2(b) and 2(c), respectively. In the serial architecture, 4 unit pumps (UPs) are connected in series, as shown in Figure 2(a).

In Figure 2(b), we assume that sunlight intensity is moderate, where $V_{\mathrm{SC}}$ becomes $2.40 \mathrm{~V}$, that is, higher than $V_{\mathrm{SC}}=1.81 \mathrm{~V}$ in weak sunlight. For the amount of $I_{\mathrm{SC}}, I_{\mathrm{SC}}$ in moderate sunlight is larger than weak sunlight, as shown in Figure 1(a). Owing to these higher $V_{\mathrm{SC}}$ and larger $I_{\mathrm{SC}}$ in the moderate sunlight condition than the weak condition, we can consider the parallel-serial architecture more suitable than the serial in Figure 2(a) in delivering larger output current to the load. In the parallel-serial architecture, two UPs are in parallel and two parallel-connected pumps are in series, as shown in Figure 2(b). Figure 2(c) shows the parallel architecture that has four UPs in parallel. This parallel architecture can be thought to be suitable to deliver the largest amount of output current to the load than the serial and parallel-serial architectures in Figures 2(a) and 2(b), respectively.

As explained just earlier, RCP circuit can maximize the amount of output current, by choosing the most suitable architecture among the serial, parallel-serial, and parallel architectures at a given sunlight condition [9]. However, performance of RCP circuit can be improved more by adding variable pumping frequency (VPF) scheme to the reconfigurable architecture. By merging the VPF scheme with the reconfigurable architecture, the charge pump which is proposed in this paper can track MPPs better than charge pumps only with the reconfigurable architecture.

\section{Variable Pumping Frequency Scheme with Reconfigurable Charge Pump's Architecture}

Figures 3(a), 3(b), and 3(c) show the amounts of output current of RCP circuit for weak, moderate, and strong sunlight, respectively, with varying the pumping frequency. In Figure 3(a) with weak sunlight intensity from $1.5 \mathrm{~mA}$ to $2.5 \mathrm{~mA}$, the pumping frequency is varied from $1 \mathrm{MHz}$ to $30 \mathrm{MHz}$ to find the best pumping frequency at which the charge pump can deliver the largest amount of output current [10]. From Figure 3(a), it is indicated that the MPP is found around $f_{\text {PUMP }}=2.5 \mathrm{MHz}$ for the sunlight intensity as low as $1.5 \mathrm{~mA}$. If the sunlight intensity becomes $2 \mathrm{~mA}$, the best 

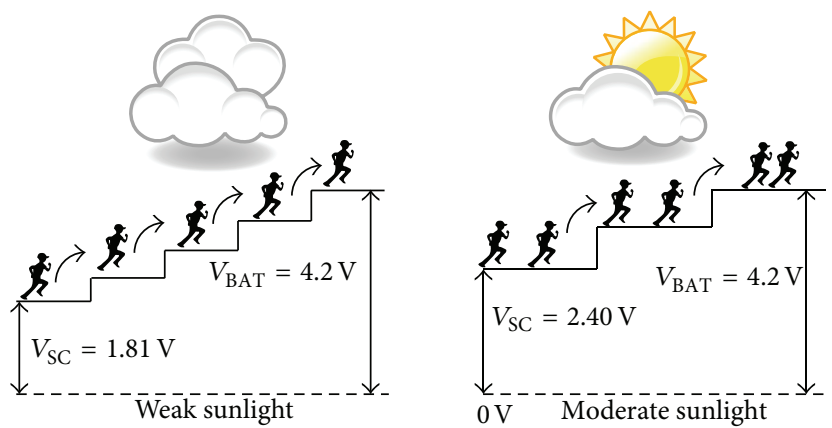

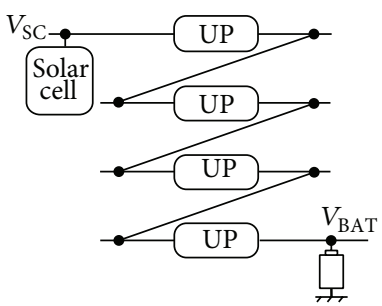

(a)

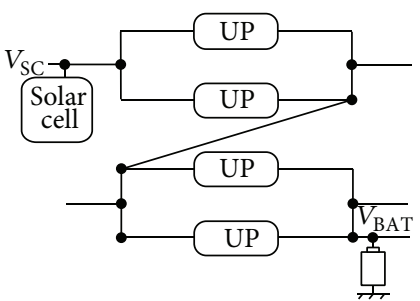

(b)
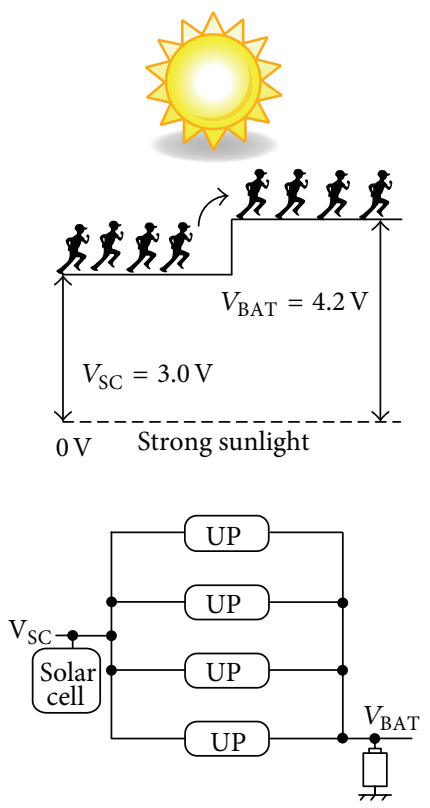

(c)

FIGURE 2: (a) Conceptual diagram of operation of RCP circuit and the serial architecture in weak sunlight condition, (b) conceptual diagram of operation of RCP circuit and the parallel-serial architecture in moderate sunlight condition, and (c) conceptual diagram of operation of RCP circuit and the parallel architecture in strong sunlight condition.

pumping frequency of delivering the maximum power to the load moves to around $2.75 \mathrm{MHz}$. For the light intensity as much as $2.5 \mathrm{~mA}$ in Figure 3(a), the charge pump can be the best in harvesting solar energy around $f_{\mathrm{PUMP}}=3 \mathrm{MHz}$. Here we can know that pumping frequency for MPP becomes higher with increasing the sunlight intensity.

Similarly, with moderate sunlight, we varied the pumping frequency to find the MPPs, where the largest amount of power can be harvested from environmental solar energy. For the moderate sunlight intensities of $3 \mathrm{~mA}, 9 \mathrm{~mA}$, and
$15 \mathrm{~mA}$, respectively, in Figure 3(b), the pumping frequencies of MPPs are found at $3 \mathrm{MHz}, 7 \mathrm{MHz}$, and $9 \mathrm{MHz}$, respectively. Figure 3(c) shows the amounts of output current with strong sunlight intensities such as $18 \mathrm{~mA}, 38 \mathrm{~mA}$, and $58 \mathrm{~mA}$. The circuit simulation indicates that the pumping frequencies of $9 \mathrm{MHz}, 15 \mathrm{MHz}$, and $23 \mathrm{MHz}$ can deliver the maximum amounts of output current to the load for the sunlight intensities of $18 \mathrm{~mA}, 38 \mathrm{~mA}$, and $58 \mathrm{~mA}$, respectively.

From Figures 3(a), 3(b), and 3(c), we can know that the VPF scheme that is merged with the reconfigurable 


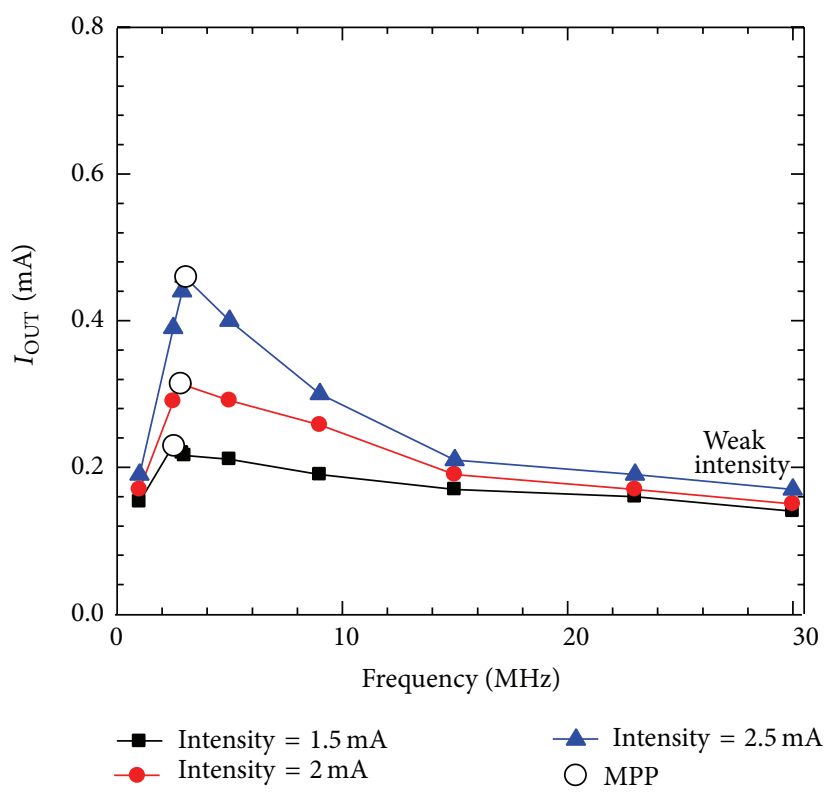

(a)

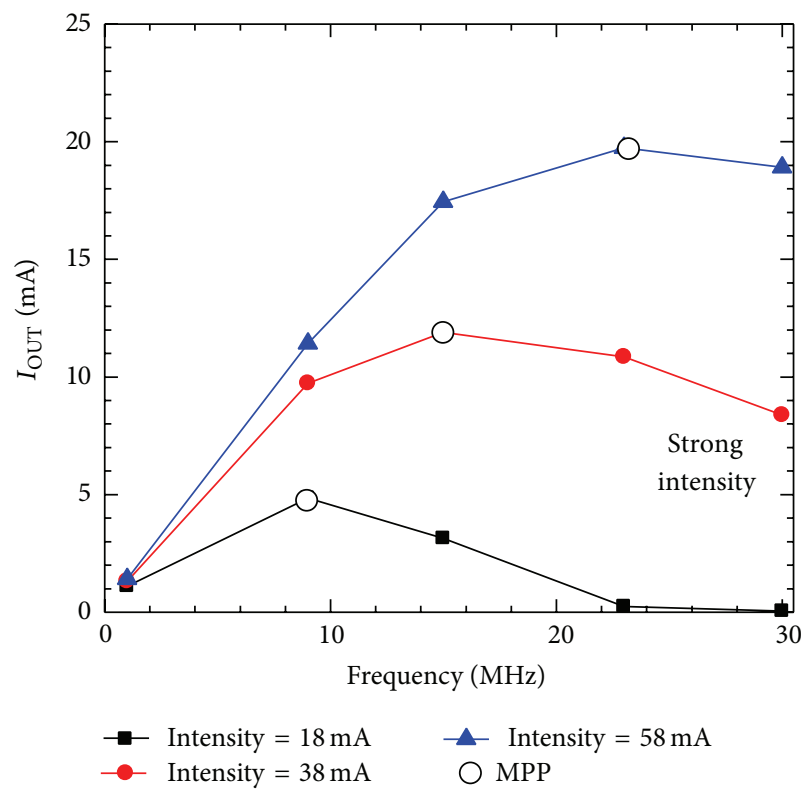

(c)

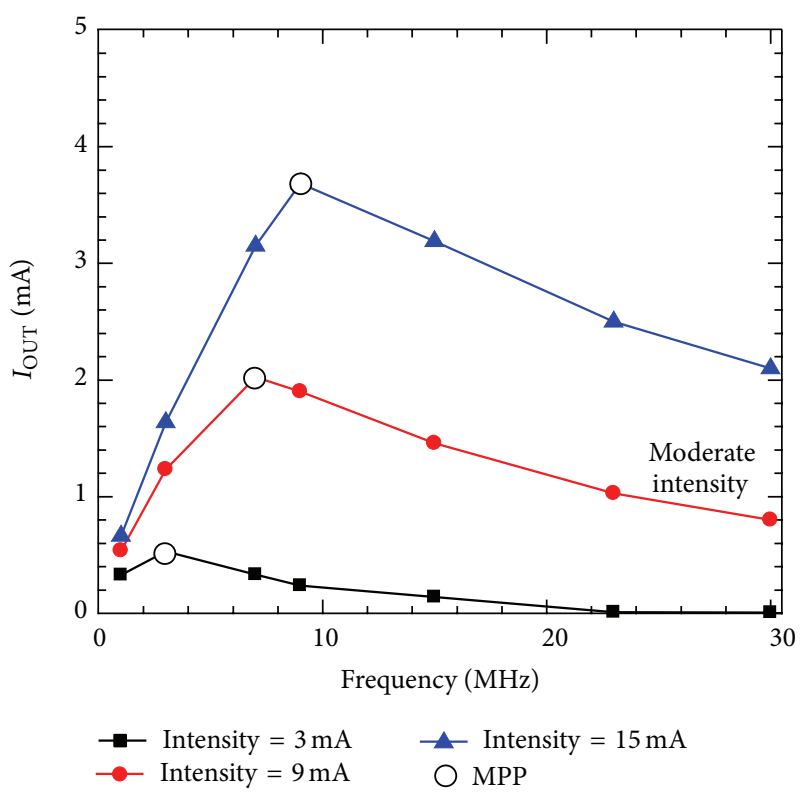

(b)

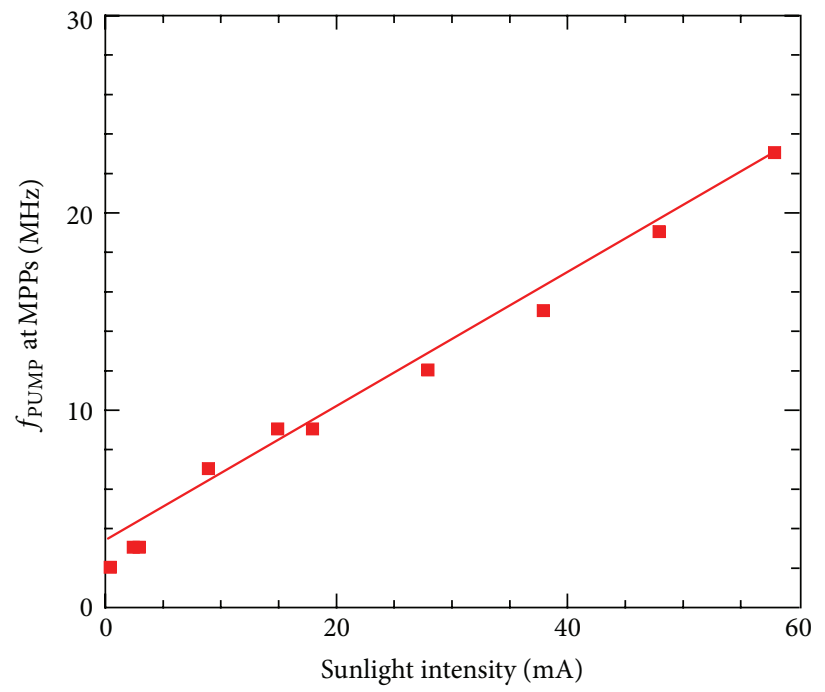

(d)

FIGURE 3: (a) Output current of RCP circuit in weak sunlight intensities with varying pumping frequency. (b) Output current in moderate sunlight with varying pumping frequency. (c) Output current in strong sunlight with varying pumping frequency. (d) The pumping frequencies at MPPs with varying sunlight intensity from $1.5 \mathrm{~mA}$ to $58 \mathrm{~mA}$.

architecture can harvest more power from environment than the charge pump circuit with only the fixed pumping frequency. Figure 3(d) shows the pumping frequencies at MPPs with varying sunlight intensity from $1.5 \mathrm{~mA}$ to $58 \mathrm{~mA}$ that includes weak, moderate, and strong sunlight intensities. From Figure 3(d), it is indicated that the pumping frequency as low as $2.5 \mathrm{MHz}$ for the sunlight intensity $=1.5 \mathrm{~mA}$ can harvest the largest amount of power from solar energy. If the sunlight intensity becomes $28 \mathrm{~mA}$, the frequency at MPP is increased to $12 \mathrm{MHz}$. For the largest sunlight intensity as large as $58 \mathrm{~mA}$, the best frequency for delivering the maximum amount of output current can be found around $23 \mathrm{MHz}$.

Figure 4 compares output currents of RCP circuit with the fixed pumping frequencies of $f_{\mathrm{PUMP}}=7 \mathrm{MHz}$ and $f_{\mathrm{PUMP}}=$ $15 \mathrm{MHz}$ and the found MPPs that are shown in Figures 3(a), $3(\mathrm{~b})$, and $3(\mathrm{c})$. Here the fixed pumping frequency of $f_{\mathrm{PUMP}}=$ $7 \mathrm{MHz}$ is decided by the MPP of moderate sunlight intensity as low as $9 \mathrm{~mA}$. If we fix pumping frequency of RCP circuit by $7 \mathrm{MHz}$, the amounts of output current at $f_{\mathrm{PUMP}}=7 \mathrm{MHz}$ are very similar with the maximum output currents in moderate 


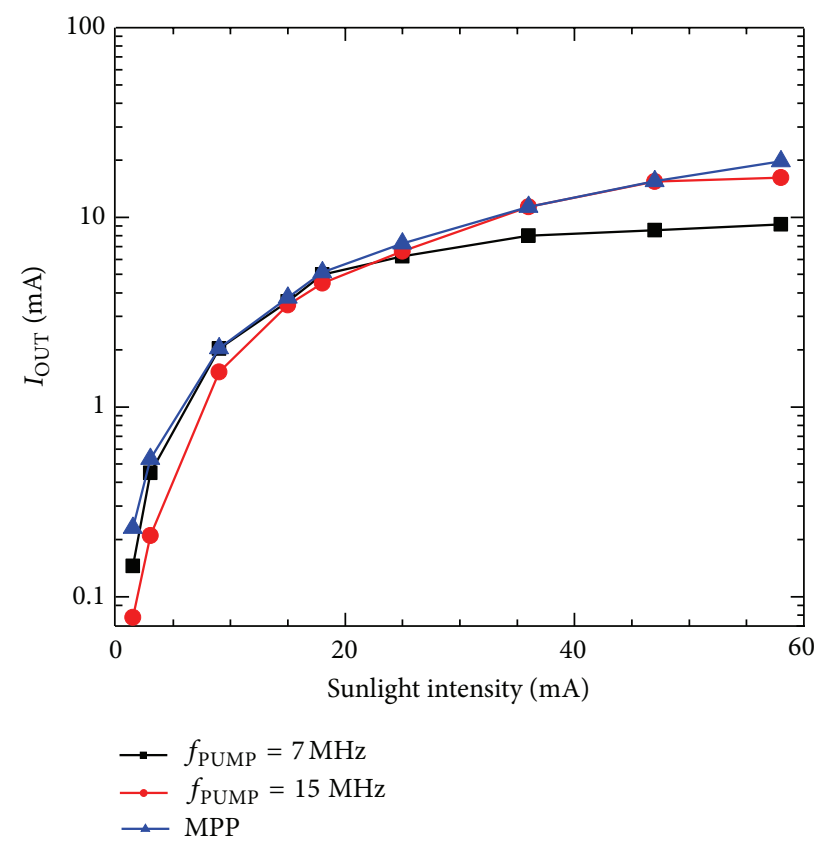

FIGURE 4: Comparison of output currents of RCP circuit with the fixed pumping frequencies $\left(f_{\mathrm{PUMP}}=7 \mathrm{MHz}\right.$ and $\left.f_{\mathrm{PUMP}}=15 \mathrm{MHz}\right)$ and MPPs that are shown in Figures 3(a), 3(b), and 3(c).

sunlight intensities from $2.5 \mathrm{~mA}$ to $15.5 \mathrm{~mA}$. On the contrary, if the intensity is increased stronger than $15.5 \mathrm{~mA}$, the output current of RCP circuit with $f_{\mathrm{PUMP}}=7 \mathrm{MHz}$ begins to deliver much smaller output current than MPPs. For the fixed pumping frequency of $15 \mathrm{MHz}$, the amounts of output current with $f_{\mathrm{PUMP}}=15 \mathrm{MHz}$ are very comparable to the MPPs in strong sunlight intensities from $15.5 \mathrm{~mA}$ to $58 \mathrm{~mA}$. However, for lighter intensities, the fixed pumping frequency of $15 \mathrm{MHz}$ cannot give the output current as much as MPPs.

From Figure 4, we can know that no fixed frequency can have the amounts of output current that are similar with the MPPs in the entire range of sunlight intensity. Thus, we need to propose the VPF scheme that can be added to the previous RCP circuit, where the pumping frequency can be changed according to a given sunlight condition to deliver the largest amount of output current to the load at this given intensity.

To give more analytical explanation on relationship of MPP and $f_{\text {PUMP }}$, we can start from the following equation of the input current of RCP circuit, $I_{\mathrm{IN}}[10]$ :

$$
I_{\mathrm{IN}}=\frac{1}{\eta} I_{\mathrm{OUT}}+\alpha_{1} f_{\mathrm{PUMP}}
$$

Here, $\eta$ is the ideal current efficiency of RCP circuit and $\alpha_{1}$ is the proportional coefficient between the charge pump's input current and the pumping frequency that accounts for switching loss and reverse current of RCP circuit that depend on the pumping frequency [10]. In this paper, we can think that the solar cell delivers the input current to RCP circuit with the additional control circuit which is needed to adjust the architecture and pumping frequency according to a sunlight variation. The solar cell's current can be expressed with [10]

$$
I_{\mathrm{SC}}=I_{\mathrm{IN}}+I_{\mathrm{DC}}+\alpha_{2} f_{\mathrm{PUMP}} .
$$

Here $I_{\mathrm{DC}}$ means the static current which is consumed in RCP circuit and the controller circuit. $\alpha_{2}$ means the switching loss in the additional control circuit. By combining (1) and (2), we can calculate $I_{\text {OUT }}$ with

$$
I_{\mathrm{OUT}}=\eta\left(I_{\mathrm{SC}}-I_{\mathrm{DC}}-\left(\alpha_{1}+\alpha_{2}\right) f_{\mathrm{PUMP}}\right) .
$$

As we increase $f_{\mathrm{PUMP}}, I_{\mathrm{SC}}$ becomes larger; thus, we can have more $I_{\mathrm{OUT}}$ at the load. However, once $I_{\mathrm{SC}}$ starts to saturate, $I_{\text {OUT }}$ should be lowered because of $-\left(\alpha_{1}+\alpha_{2}\right) f_{\mathrm{PUMP}}$ in spite of increasing $f_{\text {PUMP }}$. Thus, we can have an optimum pumping frequency for a given sunlight condition, at which the charge pump circuit can deliver the largest amount of output current to the load.

From (3), we can extract three parameters of $\eta, I_{\mathrm{DC}}$, and $\alpha_{1}+\alpha_{2}$ using the least-square fitting method [11]. For the strong sunlight condition with the parallel architecture, the extracted values of $\eta, I_{\mathrm{DC}}$, and $\alpha_{1}+\alpha_{2}$ are $0.607,3.89 \mu \mathrm{A}$, and $0.744 \mathrm{~mA} / \mathrm{Hz}$, respectively.

If we know the value of $\eta$, we can rewrite (3) as follows:

$$
\eta I_{\mathrm{SC}}-I_{\mathrm{OUT}}=\eta\left(\alpha_{1}+\alpha_{2}\right) f_{\mathrm{PUMP}}+\eta I_{\mathrm{DC}} .
$$

Equation (4) is shown in Figure 5, where the symbols represent the simulated values of $\eta I_{\mathrm{SC}}-I_{\mathrm{OUT}}$ and the line is calculated with $\eta\left(\alpha_{1}+\alpha_{2}\right) f_{\mathrm{PUMP}}+\eta I_{\mathrm{DC}}$ using the values of $\eta$, $I_{\mathrm{DC}}$, and $\alpha_{1}+\alpha_{2}$ that are stated just earlier, as we increase the pumping frequency from $1 \mathrm{MHz}$ to $30 \mathrm{MHz}$. From Figure 5, we can see that the simulated values of $\eta I_{\mathrm{SC}}-I_{\mathrm{OUT}}$ are in good agreement with the calculated values of $\eta\left(\alpha_{1}+\alpha_{2}\right) f_{\mathrm{PUMP}}+$ $\eta I_{\mathrm{DC}}$. The good agreement between the simulation and the model in (4) indicates that (3) can describe the charge pump's output current well.

\section{New Reconfigurable Charge Pump Circuit Merged with Variable Pumping Frequency Scheme}

In this session, a new RCP circuit is proposed, where the reconfigurable architecture is merged with the VPF scheme, as shown in Figure 6(a). Here the solar cell is connected to the voltage divider with the division ratio as much as 0.5 . Thus we can have a voltage as high as $0.5 \cdot V_{\mathrm{SC}}$ using the divider. By applying the sampling frequency, $f_{\text {SAMPLE }}$ which is much slower than $f_{\mathrm{PUMP}}$ for the open-circuit condition, the divided voltage, $0.5 \cdot V_{\mathrm{OC}}$ can be sampled by the sample and hold circuit (S\&H). In this paper, $f_{\text {SAMPLE }}$ used in the simulation is as slow as $1 \mathrm{KHz}$ [9]. Comparing $f_{\text {SAMPLE }}$ with $f_{\text {PUMP }}, f_{\text {SAMPLE }}$ is $\sim 1000 \mathrm{x}$ slower than $f_{\mathrm{PUMP}}$; thus, the timing overhead due to the sampling of the open-circuit voltage can be ignored. Here it should be noted that $0.5 \cdot V_{\mathrm{OC}}$ is half the sampled solar cell's voltage with the open-circuit condition. The S\&H's output, $0.5 \cdot V_{\mathrm{OC}}$, goes into the architecture reconfiguration block (ARB), where the charge pump's architecture can be 


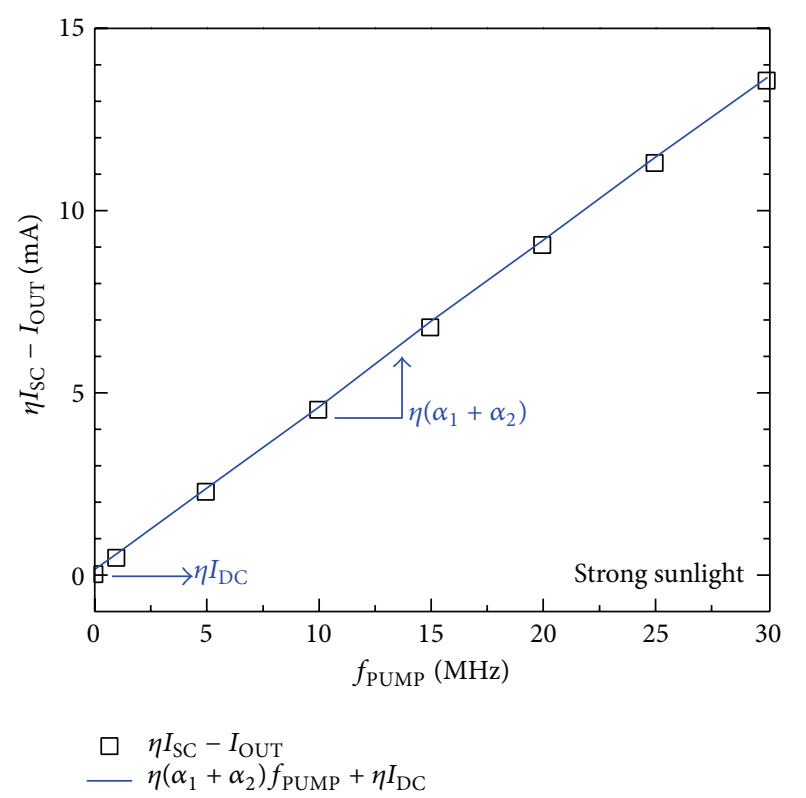

FIGURE 5: $\eta I_{\text {SC }}-I_{\text {OUT }}$ versus $\eta\left(\alpha_{1}+\alpha_{2}\right) f_{\text {PUMP }}+\eta I_{\text {DC }}$ with increasing $f_{\text {PUMP. }}$. The good agreement between $\eta I_{\mathrm{SC}}-I_{\mathrm{OUT}}$ and $\eta\left(\alpha_{1}+\right.$ $\left.\alpha_{2}\right) f_{\mathrm{PUMP}}+\eta I_{\mathrm{DC}}$ indicates that (3) can describe the charge pump's output current driven by the solar cell well.

reconfigured by controlling the reconfiguration switches [9]. As well explained in [9], three modes of RCP circuit are available in Figure 6(a). They are the serial, parallel-serial, and parallel architecture, respectively. The sampled $0.5 \cdot V_{\mathrm{OC}}$ is compared with the predetermined reference voltages such as $V_{\text {REF_PM }}$ and $V_{\text {REF_SM }}$; thereby, the charge pump architecture can be reconfigured. $\mathrm{C} 1$ and $\mathrm{C} 2$ are the comparators for the architecture reconfiguration. If $0.5 \cdot V_{\mathrm{OC}}<V_{\text {REF_SM }}$, the architecture is changed to the serial mode [9]. If $0.5 \cdot V_{\mathrm{OC}}$ is between $V_{\text {REF_SM }}$ and $V_{\text {REF_PM }}$, the circuit is reconfigured by the parallel-serial mode. When $0.5 \cdot V_{\mathrm{OC}}$ is larger than $V_{\mathrm{REF} \text {.PM }}$, the mode is fixed by the parallel architecture.

The $0.5 \cdot V_{\mathrm{OC}}$, that is, a signal before the $\mathrm{S} \& \mathrm{H}$ circuit in Figure 6(a) goes into the variable frequency block (VFB), where the solar cell's voltage, $V_{\mathrm{SC}}$ is compared with $0.5 \cdot k \cdot V_{\mathrm{OC}}-\Delta$ and $0.5 \cdot k \cdot V_{\mathrm{OC}}+\Delta$ to decide the optimum pumping frequency, at which the maximum solar power can be harvested from environment. Here $k$ is a fractional constant of $V_{\mathrm{OC}}$ and the value used in this simulation is 0.8 . It is well known that the solar cell can deliver the maximum power to the load, when $V_{\mathrm{SC}}$ is close to a fractional opencircuit voltage, $k \cdot V_{\mathrm{OC}}$, even though $k$ can be different for various kinds of solar cells [12]. If the optimum pumping frequency is found, the voltage-controlled oscillator (VCO) applies the obtained $f_{\mathrm{PUMP}}$ of MPP to RCP circuit. The schematic of UP circuit is shown in the inset of Figure 6(a), where $M_{1}$ and $M_{3}$ are the precharging NMOSFETs and $M_{2}$ and $M_{4}$ are the transferring PMOSFETs. The width and length of $M_{1}$ and $M_{3}$ are $120 \mu \mathrm{m}$ and $0.35 \mu \mathrm{m}$, respectively. The width and length of $M_{2}$ and $M_{4}$ are $240 \mu \mathrm{m}$ and $0.35 \mu \mathrm{m}$, respectively. The pumping capacitors are $\mathrm{CAP}_{1}$ and $\mathrm{CAP}_{2}$ in Figure 6(a). The capacitance used in the simulation is $50 \mathrm{pF}$.
CLKB and CLK are the two-phase clocking signals that enter $\mathrm{CAP}_{1}$ and $\mathrm{CAP}_{2}$, respectively. Figure 6(b) shows the detailed schematic of VFB with VCO circuit. For VCO circuit, the general scheme which is based on simple ring oscillator is used in this paper [13].

Figure 6(c) shows the voltage and current waveforms in Figure 6(a). If $0.5 \cdot V_{\mathrm{SC}}$ is between $0.5 \cdot k \cdot V_{\mathrm{OC}}-\Delta$ and $0.5 \cdot k \cdot V_{\mathrm{OC}}+\Delta$, the charge pump (CP) in VFB keeps its output voltage, $V_{C}$, which controls the VCO. If $V_{C}$ is not changed, the VCO's output frequency, $f_{\mathrm{PUMP}}$, is not changed, too. Now let us explain the closed-loop operation of VPF scheme in Figure 6(a) more in detail, as shown in Figure 6(c). First, we can assume that $0.5 \cdot V_{\mathrm{SC}}$ is larger than $0.5 \cdot k \cdot V_{\mathrm{OC}}+\Delta$. At this time, the two comparators of $C_{3}$ and $C_{4}$ can give UP signal to $\mathrm{CP}$ and $\mathrm{VCO}$ in Figure 6(a) to increase the pumping frequency higher. As the pumping frequency becomes higher, $0.5 \cdot V_{\mathrm{SC}}$ becomes lower. If $0.5 \cdot V_{\mathrm{SC}}$ is between $0.5 \cdot k \cdot V_{\mathrm{OC}}-\Delta$ and $0.5 \cdot k \cdot V_{\mathrm{OC}}+\Delta$, the two comparators of $C_{3}$ and $C_{4}$ give STOP signal to $\mathrm{CP}$ and VCO; thereby, the pumping frequency is stabilized. If $0.5 \cdot V_{\mathrm{SC}}$ is lower than $0.5 \cdot k \cdot V_{\mathrm{OC}}-\Delta$, the comparators $C_{3}$ and $C_{4}$ generate DOWN signal to $\mathrm{CP}$ and VCO; thus, the pumping frequency becomes lower and $0.5 \cdot V_{\mathrm{SC}}$ can be raised little by little until $0.5 \cdot V_{\mathrm{SC}}$ is between $0.5 \cdot k \cdot V_{\mathrm{OC}}-\Delta$ and $0.5 \cdot k \cdot V_{\mathrm{OC}}+\Delta$.

\section{Simulation Results and Layout}

The proposed circuit is verified by SPECTRE circuit simulation which is provided by Cadence Inc. The SPECTRE simulation model was obtained from Magna $0.35 \mu \mathrm{m}$ CMOS process technology. Figure 7(a) compares the percentage amounts of output current among 3 schemes of the reconfigurable charge pump circuit, with respect to MPPs. Here " $f_{\text {PUMP }}=7 \mathrm{MHz}$ " in Figure 7 (a) means that the output current of RCP circuit is obtained when the pumping frequency is fixed at $7 \mathrm{MHz}$. For " $f_{\mathrm{PUMP}}=7 \mathrm{MHz}$ ", even though the sunlight condition is changed, the pumping frequency is fixed at $7 \mathrm{MHz}$ for all the sunlight conditions. In Figure 7(a), the fixed pumping frequency as low as $7 \mathrm{MHz}$ is decided by the fact that the charge pump circuit can deliver the maximum output current at $f_{\mathrm{PUMP}}=7 \mathrm{MHz}$ for the moderate sunlight condition of the intensity $=9 \mathrm{~mA}$, as shown in Figure 3(b). In Figure 7(a), $f_{\text {PUMP }}=15 \mathrm{MHz}$ is the output current of RCP circuit when the pumping frequency of Figure 6(a) is fixed at $15 \mathrm{MHz}$. Similarly, with " $f_{\mathrm{PUMP}}=7 \mathrm{MHz}$ ", the fixed pumping frequency as high as $15 \mathrm{MHz}$ is decided because the charge pump can have the largest output current for the strong sunlight condition of intensity $=38 \mathrm{~mA}$, as shown in Figure 3(c). In Figure 7(a), "VPF" means that the pumping frequency of the reconfigurable charge pump can be variable according to the sunlight conditions. Here the pumping frequency is decided to meet the condition where the solar cell's voltage, $0.5 V_{\mathrm{SC}}$, should be between $0.5 \cdot k \cdot V_{\mathrm{OC}}-\Delta$ and $0.5 \cdot k \cdot V_{\mathrm{OC}}+\Delta$. This variable frequency for each sunlight condition can be tracked by the circuit shown in Figures 6(a) and 6(b). In Figure 7(a), "MPP" means the amounts of output current of RCP circuit at the maximum power points for various sunlight conditions. The maximum amounts of $I_{\mathrm{OUT}}$ 


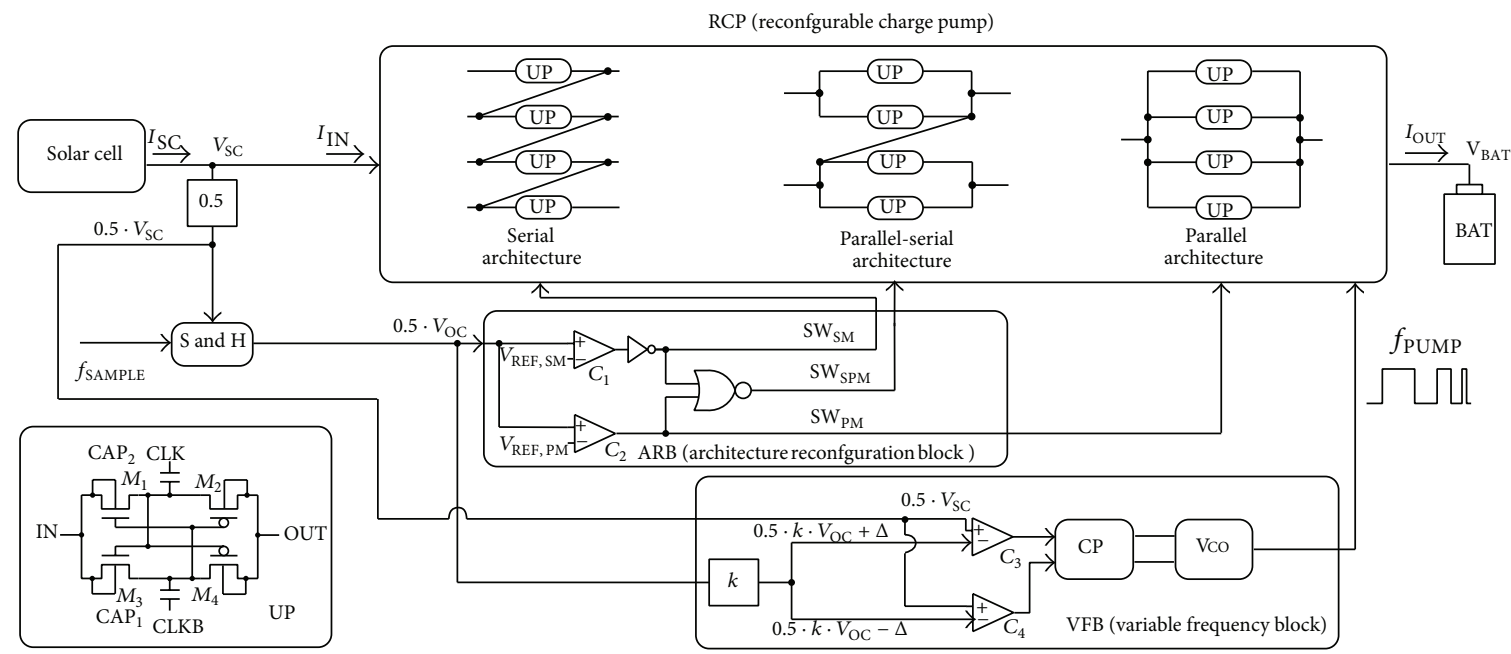

(a)

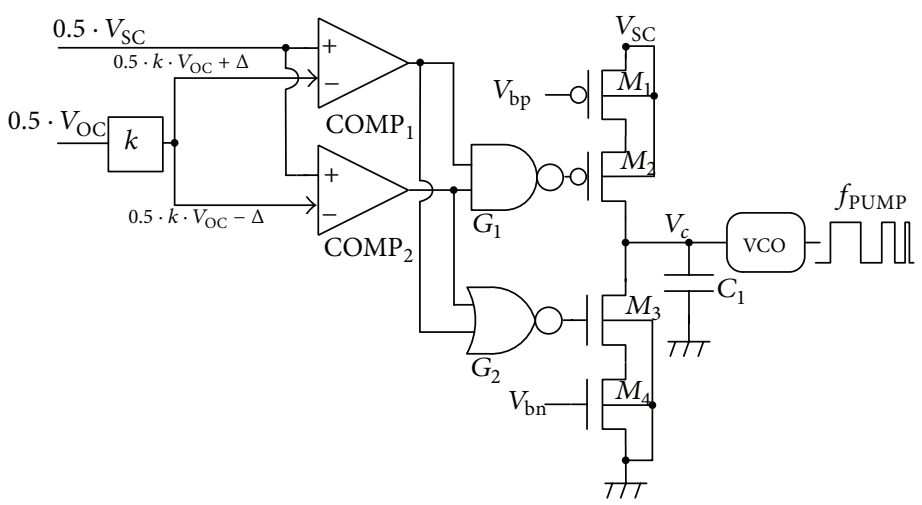

(b)

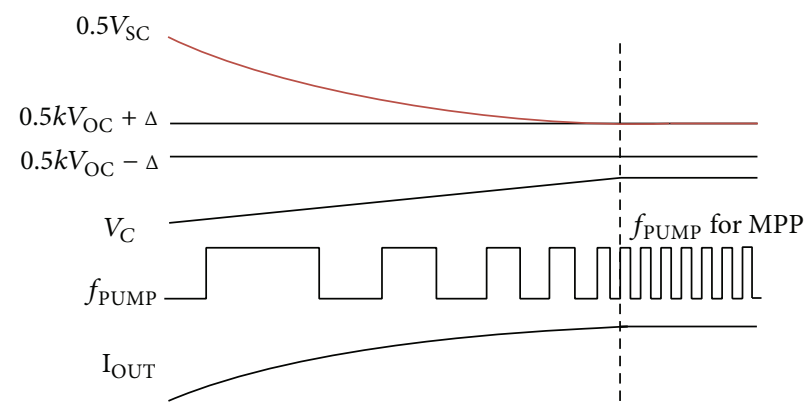

(c)

Figure 6: (a) Block diagram of the new RCP circuit that is proposed in this paper. (b) Circuit schematic of the VFB. (c) Voltage and current waveforms of the VFB.

for various sunlight conditions are obtained by the circuit simulation of the charge pump with varying the pumping frequency from $1 \mathrm{MHz}$ to $30 \mathrm{MHz}$.

From Figure $7(\mathrm{a})$, " $f_{\text {PUMP }}=7 \mathrm{MHz}$ " shows $99.6 \%$ of the maximum output current, at the moderate sunlight condition. However, " $f_{\text {PUMP }}=7 \mathrm{MHz}$ " shows only $63.1 \%$ and $48.9 \%$ at the weak and strong sunlight conditions, respectively. This fact tells us that $f_{\mathrm{PUMP}}$ as low as $7 \mathrm{MHz}$ can deliver the largest amount of $I_{\mathrm{OUT}}$ in only moderate sunlight intensity compared to weak and strong sunlight intensities. If sunlight intensity becomes different from the moderate condition, percentage of $I_{\mathrm{OUT}}$ with respect to the MPP becomes much smaller. " $f_{\text {PUMP }}=15 \mathrm{MHz}$ " can deliver $99.9 \%$ of the maximum output current in strong sunlight. In spite of this large output current in strong intensity, RCP circuit at the fixed pumping frequency of $15 \mathrm{MHz}$ can generate as low as $33.8 \%$ and $74.7 \%$ of the maximum $I_{\text {OUT }}$, in the weak and moderate sunlight condition, respectively. Unlike the cases of the fixed pumping frequencies of $f_{\mathrm{PUMP}}=$ $7 \mathrm{MHz}$ and $15 \mathrm{MHz}$, the VPF scheme which is proposed in 


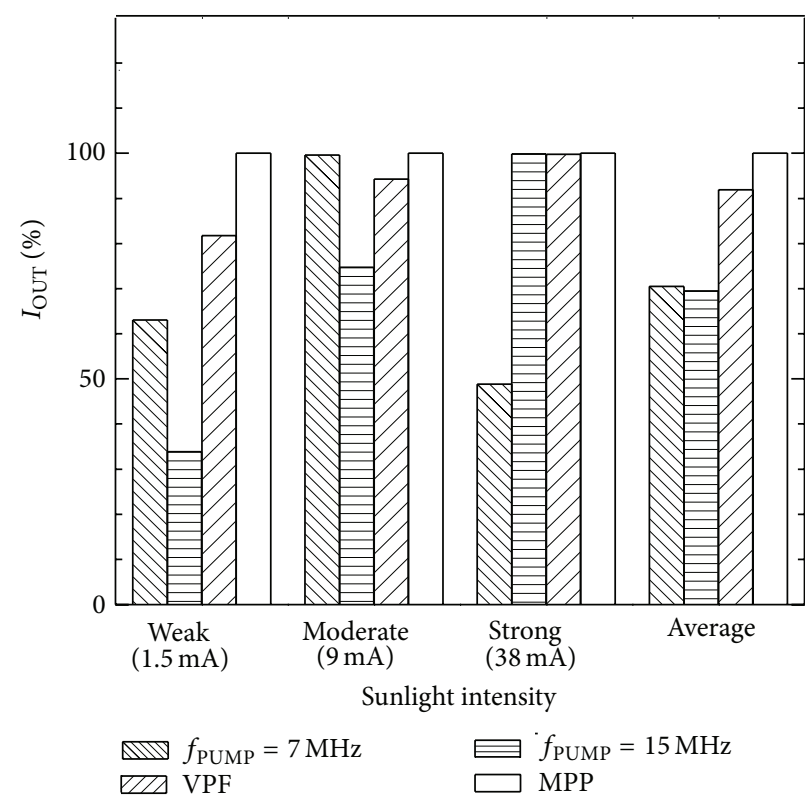

(a)

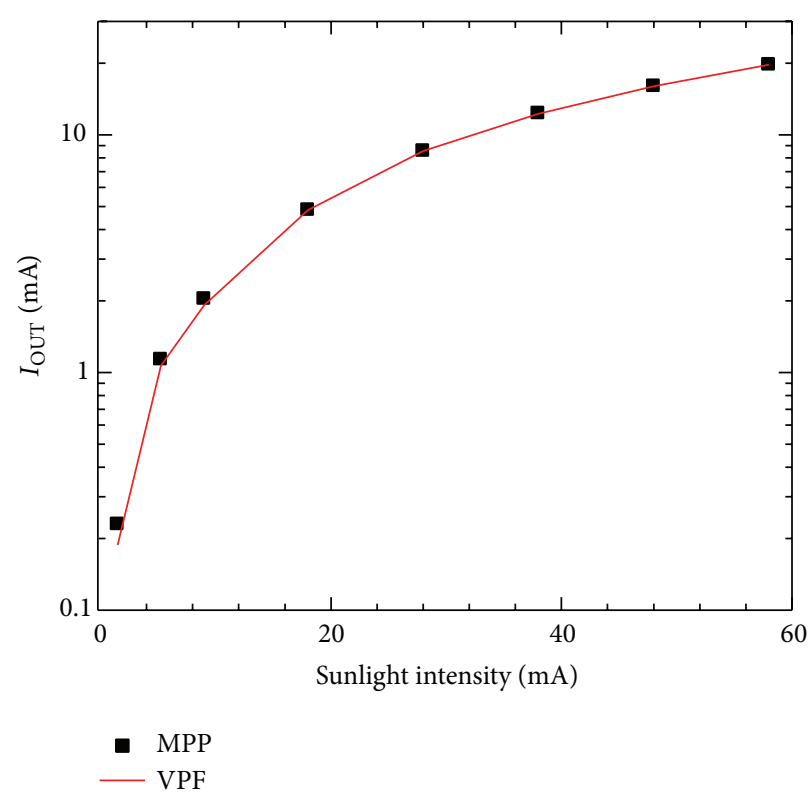

(b)

Figure 7: (a) The percentage $I_{\text {OUT }}$ for various sunlight conditions with the fixed pumping frequencies of $7 \mathrm{MHz}$ and $15 \mathrm{MHz}$ and the VPF scheme. Here MPP means the simulated maximum output currents. (b) The output current comparison between the proposed VPF scheme and the simulated maximum output currents (MPP).

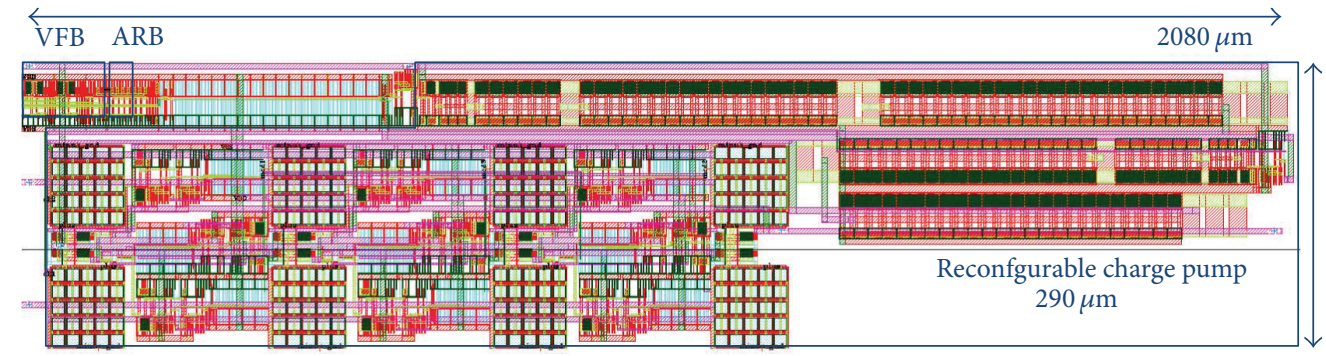

FIGURE 8: Layout of RCP circuit with the added VPF scheme.

this paper shows $91.9 \%$ of the maximum amounts of current for all the sunlight conditions from weak to strong intensity, on average. The large amounts of output current in the VPF scheme can be delivered to the load in a wide range of sunlight intensity from weak to strong. Figure 7(b) compares the VPF scheme with respect to MPPs from the sunlight intensity from $1.5 \mathrm{~mA}$ to $58 \mathrm{~mA}$. For the entire range of sunlight intensity, the VPF scheme shows that amounts of the output current are similar with MPPs that are obtained from Figures 3(a), 3(b), and $3(\mathrm{c})$.

Figure 8 shows the layout of the proposed RCP circuit with VPF scheme. The VPF scheme which is added in this paper occupies an area as small as $1.61 \%$ of the total layout area. The power overhead which is caused by the added VPF scheme is estimated only as small as $0.4 \%$ of the total power consumption, for the sunlight condition as strong as $38 \mathrm{~mA}$. If the sunlight conditions become as weak as $9 \mathrm{~mA}$, the power overhead is increased to $1.7 \%$. This is due to the fact that the solar cell's current becomes smaller with decreasing the sunlight intensity, while the amount of power consumption of the VPF scheme is changed very little. Thus the percentage power overhead becomes worse with decreasing sunlight intensity.

\section{Conclusion}

In this paper, the VPF scheme was proposed to be merged with the previous RCP circuit that can change its architecture according to a given sunlight condition. Merging the VPF scheme with the architecture reconfiguration can improve the percentage $I_{\text {OUT }}$ better by $21.4 \%$ and $22.4 \%$ than the fixed pumping frequencies of $7 \mathrm{MHz}$ and $15 \mathrm{MHz}$, respectively. Comparing the VPF scheme with the available maximum output currents, the VPF can deliver $91.9 \%$ of the maximum $I_{\mathrm{OUT}}$ to the load on average. In terms of the power and area overheads, the VPF scheme proposed in this paper consumes the power by $0.4 \%$ of the total power consumption and occupies the layout area by $1.61 \%$ of the total layout area. 


\section{Conflict of Interests}

The authors declare that there is no conflict of interests regarding the publication of this paper.

\section{Acknowledgments}

The work was financially supported by NRF-2011-220D00089, NRF-2011-0030228, NRF-2013K1A3A1A25038533, NRF-2013R1A1A2A10064812, and BK Plus with the Educational Research Team for Creative Engineers on MaterialDevice-Circuit Co-Design (Grant no. 22A20130000042), funded by the National Research Foundation of Korea (NRF) and the Industrial Strategic Technology Development Program funded by the Ministry of Trade, Industry and Energy (MOTIE, Korea) (10039239). The CAD tools were supported by IC Design Education Center (IDEC), Daejeon, Korea.

\section{References}

[1] J. Kim, J. Kim, and C. Kim, "A regulated charge pump with a low-power integrated optimum power point tracking algorithm for indoor solar energy harvesting," IEEE Transactions on Circuits and Systems II: Express Briefs, vol. 58, no. 12, pp. 802806, 2011.

[2] C. Lu, S. P. Park, V. Raghunathan, and K. Roy, "Low-overhead maximum power point tracking for micro-scale solar energy harvesting systems," in Proceedings of the 25th International Conference on VLSI Design (VLSID '12), pp. 215-220, January 2012.

[3] J. Schmid, T. Gaedeke, T. Scheibe, and W. Stork, "Improving energy efficiency for small-scale solar energy harvesting," in Proceedings of the European Conference on Smart Objects, Systems and Technologies, pp. 1-9, June 2012.

[4] C. Alippi and C. Galperti, "An adaptive system for opimal solar energy harvesting in wireless sensor network nodes," IEEE Transactions on Circuits and Systems I: Regular Papers, vol. 55, no. 6, pp. 1742-1750, 2008.

[5] S. Bader and B. Oelmann, "Short-term energy storage for wireless sensor networks using solar energy harvesting," in Proceedings of the IEEE International Conference on Networking, Sensing and Control, pp. 71-76, April 2013.

[6] P. Favrat, P. Deval, and M. J. Declercq, "A high-efficiency CMOS voltage doubler," IEEE Journal of Solid-State Circuits, vol. 33, no. 3, pp. 410-416, 1998.

[7] F. Pen and T. Samaddar, Charge Pump Circuit Design, McGrawHill, New York, NY, USA, 2006.

[8] J. Nelson, The Physics of Solar Cells, Imperial College Press, London, UK, 2003.

[9] E. S. Lee, J. M. Choi, Y. S. Kim et al., "Sunlight-variationadaptive charge pump circuit with self-reconfiguration for small-scale solar energy harvesting," IEICE Electronics Express, vol. 9, no. 17, pp. 1423-1433, 2012.

[10] H. Shao, C.-Y. Tsui, and W.-H. Ki, "An inductor-less micro solar power management system design for energy harvesting applications," in Proceedings of the IEEE International Symposium on Circuits and Systems (ISCAS '07), pp. 1353-1356, May 2007.

[11] T. Kariya and H. Kurata, Generalized Least Squares, John Wiley \& Sons, New York, NY, USA, 2004.

[12] J. Ahmad, "A fractional open circuit voltage based maximum power point tracker for photovoltaic arrays," in Proceedings of the 2nd International Conference on Software Technology and Engineering (ICSTE '10), pp. 247-250, October 2010.

[13] P. E. Allen and D. R. Holberg, CMOS Analog Circuit Design, Oxford University, Oxford, UK, 2002. 

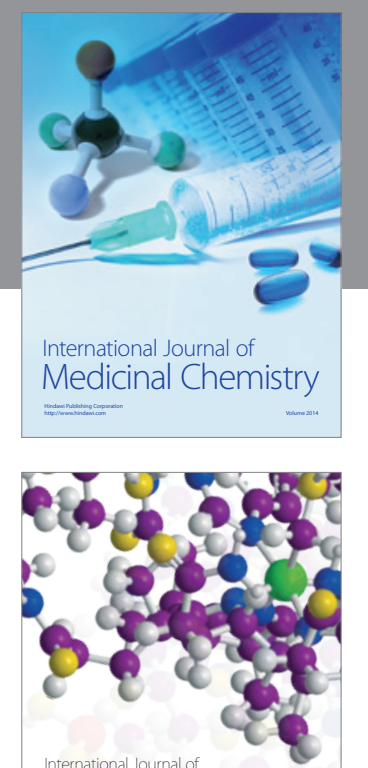

\section{Carbohydrate} Chemistry

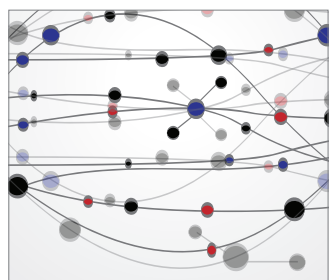

The Scientific World Journal
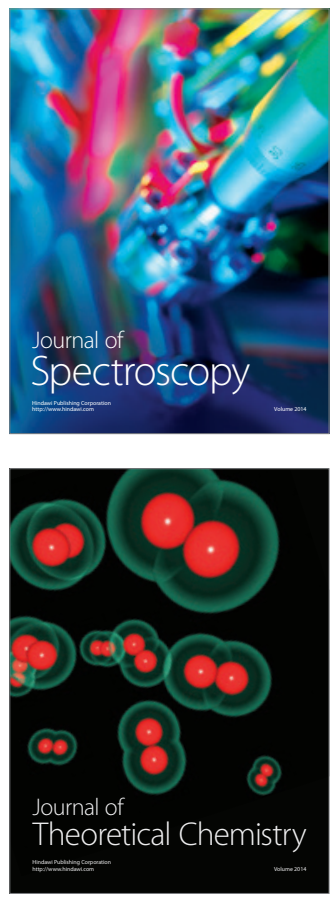
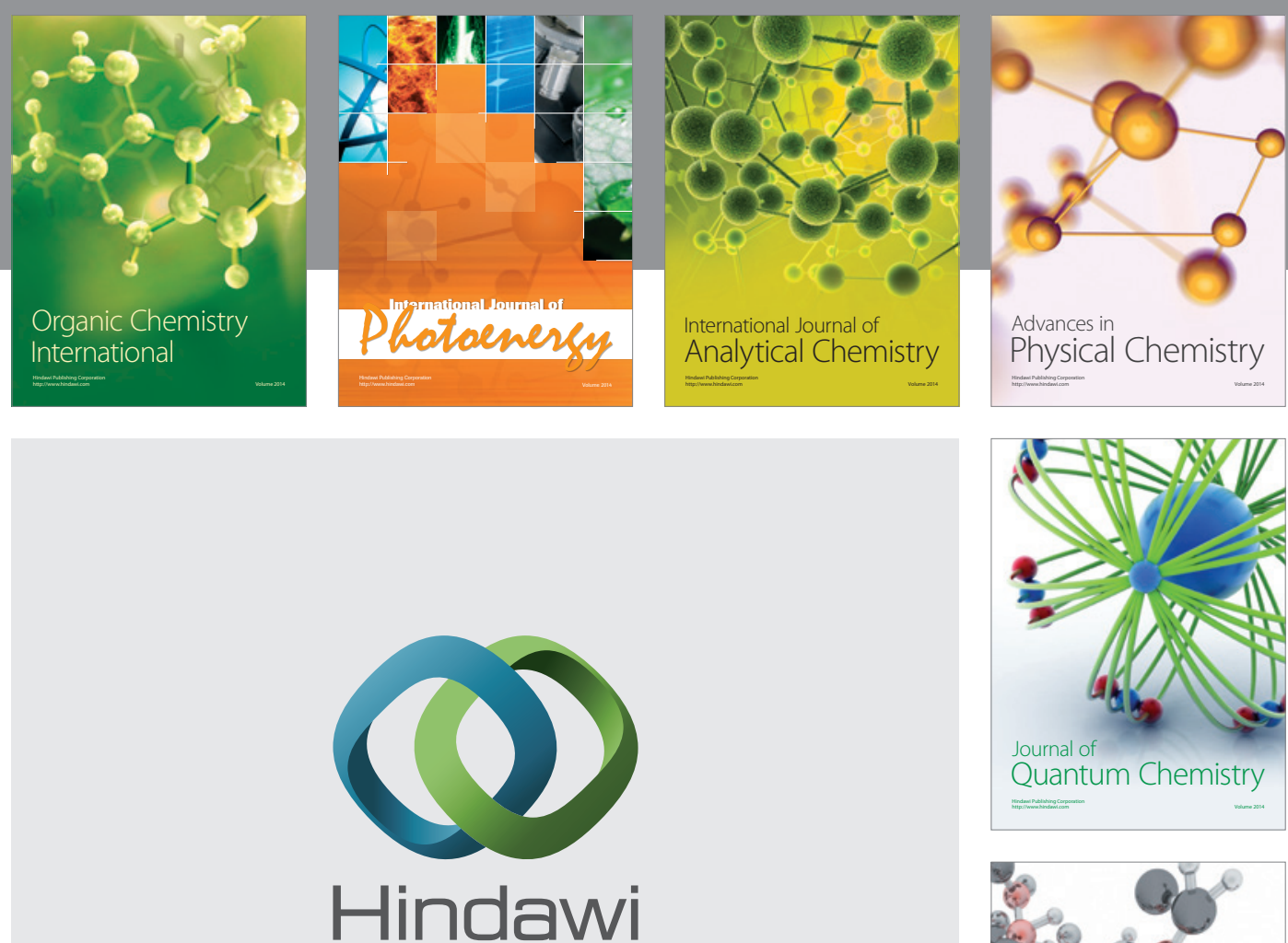

Submit your manuscripts at

http://www.hindawi.com

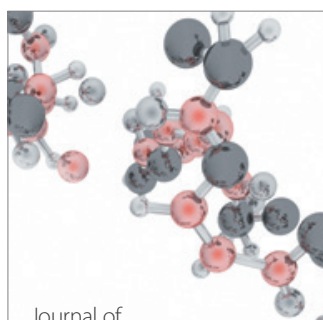

Analytical Methods

in Chemistry

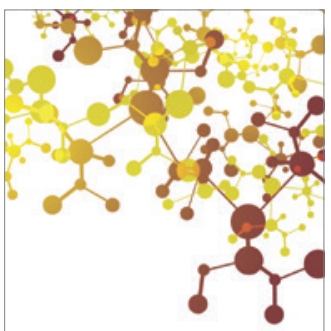

Journal of

Applied Chemistry

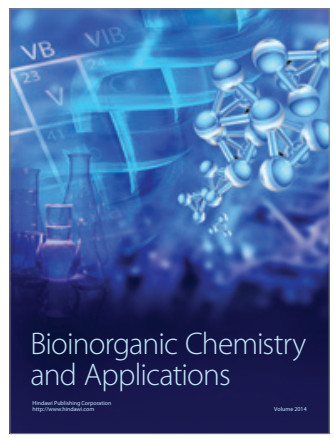

Inorganic Chemistry
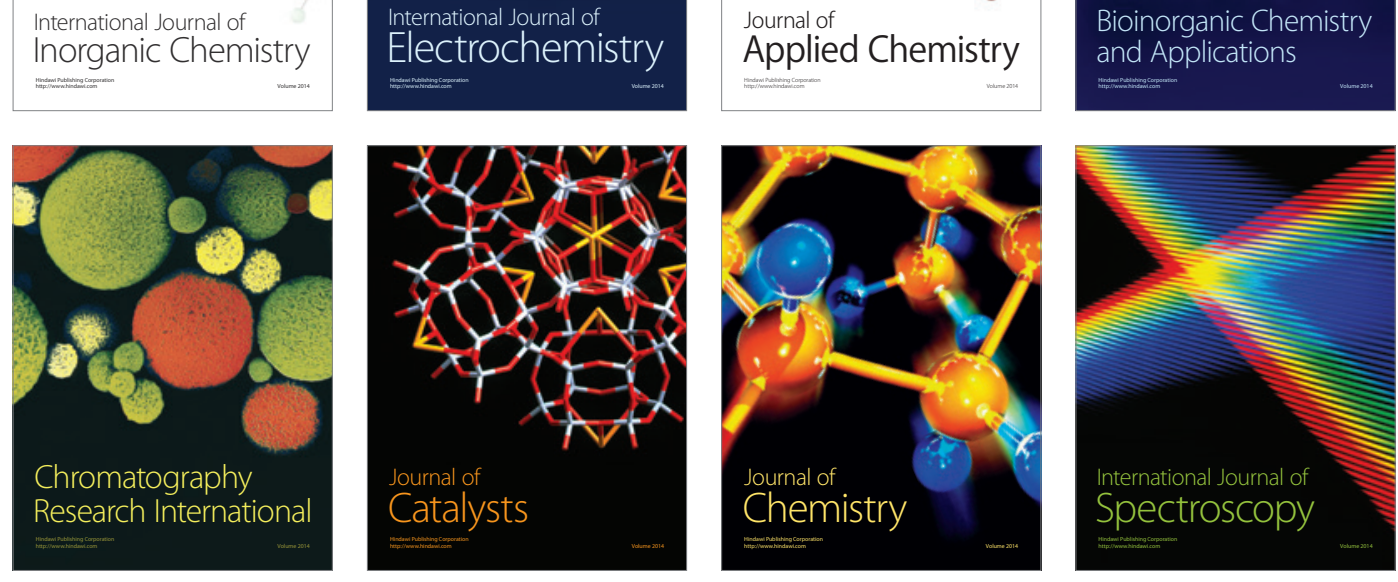